\title{
LINEAR STABILITY OF SHOCK PROFILES FOR A RATE-TYPE VISCOELASTIC SYSTEM WITH RELAXATION
}

BY

TAO LUO (Consiglio Nazionale delle Ricerche Istituto per le Applicazioni del Calcolo (M.Picone), Viale del Policlinico 137 I-00161 Roma, Italy)

AND

DENIS SERRE (UMPA (UMR 128 CNRS), Ecole Normale Supérieure de Lyon, 46, Allée d'Italie 69364, Lyon Cedex 07, France)

Abstract. We investigate the linear stability of a shock profile for a rate-type viscoelastic system with relaxation. The linear stability of shock profiles for a general initial perturbation is proved by introducing new waves through time-asymptotic expansion and using energy estimates.

1. Introduction. The purpose of this paper is to contribute to the investigation of the stability of shock profiles for a viscoelastic system with relaxation. Namely, consider the system

$$
\begin{gathered}
v_{t}-u_{x}=0 \\
u_{t}+p_{x}=0, \\
(p+E v)_{t}=-\frac{\left(p-p_{R}(v)\right)}{\tau}
\end{gathered}
$$

with the initial data

$$
v(x, 0)=v_{0}(x), \quad u(x, 0)=u_{0}(x), \quad p(x, 0)=p_{0}(x)
$$

where $v$ and $(-p)$ denote strain and stress, $u$ is related to the particle velocity, $E$ is a positive constant, called the dynamic Young's modulus, and $\tau$ is the relaxation time. The model (1.1) was proposed in [SU] to approximate the following system of conservation laws:

$$
\begin{aligned}
v_{t}-u_{x} & =0, \\
u_{t}+\left(p_{R}(v)\right)_{x} & =0 .
\end{aligned}
$$

Received February 20, 1996.

1991 Mathematics Subject Classification. Primary 35L60, 35L65.

This research was achieved while the first author visited the ENS of Lyon, supported by CNRS (France). Permanent address of first author: Institute of Mathematics, Academia Sinica, Beijing 100080, China. 
For any given shock wave $\left(s ; v^{-}, u^{-} ; v^{+}, u^{+}\right)$of (1.3) satisfying the entropy condition, it has been proved in [HL] that the system (1.1) admits a smooth travelling-wave solution:

$$
(\bar{v}, \bar{u}, \bar{p})(x, t)=(\bar{v}, \bar{u}, \bar{p})(y)
$$

with

$$
\begin{aligned}
& \lim _{y \rightarrow \pm \infty} \bar{v}(y)=v^{ \pm}, \\
& \lim _{y \rightarrow \pm \infty} \bar{u}(y)=u^{ \pm}, \\
& \lim _{y \rightarrow \pm \infty} \bar{p}(y)=p^{ \pm}=: p_{R}\left(v^{ \pm}\right)
\end{aligned}
$$

satisfying $(1.1)$, where $y=(x-s t) / \tau$. We call $(\bar{v}, \bar{u}, \bar{p})$ the shock profile.

The nonlinear stability of the shock profiles for the system (1.1) has been proved by Hsiao and Luo in $[\mathrm{HL}]$, but there is a restriction in this paper, which says,

$$
\int\left(v_{0}(x)-\bar{v}(x)\right) d x=0, \quad \int\left(u_{0}(x)-\bar{u}(x)\right) d x=0 .
$$

Here and below an integral lacking limits of integration is understood to be an integral over the whole real line.

The purpose of this paper is to investigate the stability of shock profiles without the restriction (1.4).

The stability of elementary waves for a $2 \times 2$ relaxation model has been proved in [LI]. In this case, the corresponding equilibrium equation is a scalar equation of conservation law. Therefore, a generic perturbation of a shock profile produces only a translation. However, when we treat the system (1.1), the corresponding equilibrium system (1.3) is $2 \times 2$. A generic perturbation of a shock profile produces not only a translation but also some new waves.

It is known that the second-order expansion of (1.1) is similar to the Chapman-Enskog expansion for the Boltzmann equation, which is a $2 \times 2$ system with viscosity. Based on this fact, we construct some new waves with diffusive properties that carry the "excess mass". Our new waves are composed of two parts. The first part has the form of $\frac{1}{\sqrt{t+1}} m\left(\frac{x-\lambda_{1}(t+1)}{\sqrt{t+1}}\right) r_{1}$, while the second part has the form of $\frac{1}{t+1} m_{1}\left(\frac{x-\lambda_{1}(t+1)}{\sqrt{t+1}}\right) \vec{A}$, where $\lambda_{1}, r_{1}$ are the eigenvalue and the right eigenvector of (1.3), respectively. $\vec{A}$ is a vector to be chosen in section 2 . We require that the first part carry the "excess mass", while the second part carries the net "zero mass". To be definite, let us call the first part the diffusive wave and the second part the high-order correction.

The idea for constructing the above waves is motivated by the following time-asymptotic expansion. Suppose the shock wave belongs to the 2-family of (1.3), and suppose $(\bar{v}, \bar{u}, \bar{p})$ is the shock profile. We perform the following time-asymptotic expansion for the solution $(v, u, p)$ :

$$
\begin{aligned}
& v=\bar{v}+\frac{a}{\sqrt{t+1}} m\left(\frac{x-\lambda_{1}(t+1)}{\sqrt{t+1}}\right)+\frac{a_{1}}{t+1} m_{1}\left(\frac{x-\lambda_{1}(t+1)}{\sqrt{t+1}}\right)+\cdots, \\
& u=\bar{u}+\frac{b}{\sqrt{t+1}} m\left(\frac{x-\lambda_{1}(t+1)}{\sqrt{t+1}}\right)+\frac{b_{1}}{t+1} m_{1}\left(\frac{x-\lambda_{1}(t+1)}{\sqrt{t+1}}\right)+\cdots .
\end{aligned}
$$

The expansion of $p$ has the same form. 
There is an extensive literature on the stability of viscous shock waves; see [LIU], [SX], [GO], [MN], etc. The main difficulty in dealing with the stability of the shock profile for the system with relaxation is that the dissipation of relaxation is weaker than the viscosity. For simplicity, we investigate linear stability as the first step. We believe the idea developed here is basic to the study of nonlinear stability.

The phenomenon of relaxation is important in many physical situations. Some important progress has been made in this subject. We refer to [CE], [PI], [NA], [IN], [KM], $[\mathrm{CP}],[\mathrm{LI}],[\mathrm{CLL}],[\mathrm{X}],[\mathrm{ST}]$, and the references there.

2. Shock profile; Diffusive waves; Decomposition of the solution. In this section, we discuss the properties of the shock profile, and establish the explicit expressions of the new waves that will play a crucial role in our stability analysis. At first, we give some assumptions on the function $p_{R}(v)$ :

H1) There exists an open set $N \subset R$ such that $p_{R}^{\prime}(v)<0$ and $p_{R}^{\prime \prime}(v)>0$ for $v \in N$.

Thus (1.3) is hyperbolic and genuinely nonlinear for $v \in N$.

A discontinuity $\left(s ; v^{-}, u^{-} ; v^{+}, u^{+}\right),\left(v^{-}, v^{+} \in N\right)$ in the weak solution of $(1.3)$ is called a shock wave if

A. The Rankine-Hugoniot condition is satisfied, namely

$$
\begin{aligned}
& s\left(v^{+}-v^{-}\right)=-\left(u^{+}-u^{-}\right), \\
& s\left(u^{+}-u^{-}\right)=p_{R}\left(v^{+}\right)-p_{R}\left(v^{-}\right) .
\end{aligned}
$$

B. The entropy condition holds, namely, for any $v$ between $v^{-}$and $v^{+}$,

$$
-\frac{p_{R}(v)-p_{R}\left(v^{+}\right)}{v-v^{+}}<s^{2}<\frac{p_{R}(v)-p_{R}\left(v^{-}\right)}{v-v^{-}},
$$

for $s>0$, and (2.2) takes the inverse inequality for $s<0$.

A shock profile is a smooth travelling-wave solution for (1.1), namely, a solution in the form

$$
(\bar{v}, \bar{u}, \bar{p})(x, t)=(\bar{v}, \bar{u}, \bar{p})(y), \quad y=\frac{(x-s t)}{\tau}
$$

satisfying

$$
v( \pm \infty)=v^{ \pm}, \quad u( \pm \infty)=u^{ \pm}, \quad p( \pm \infty)=p_{R}\left(v^{ \pm}\right)=: p^{ \pm} .
$$

We assume that $\tau=1$ for simplicity. Thus, $(\bar{v}, \bar{u}, \bar{p})$ satisfies the following equations:

$$
\begin{aligned}
-s \bar{v}^{\prime}-\bar{u}^{\prime} & =0, \\
-s \bar{u}^{\prime}+\bar{p}^{\prime} & =0, \\
s(\bar{p}+E \bar{v})^{\prime} & =\bar{p}-\bar{p}_{R}(\bar{v}) .
\end{aligned}
$$

It is proved in [HL] that the system (1.1) admits a smooth travelling-wave solution (shock profile) under the following subcharacteristic condition:

$$
-\sqrt{E}<s<\sqrt{E} .
$$

Moreover, the shock profile is unique up to a shift of $y$. 
To be definite, we assume $s>0$ and then $v^{+}>v^{-}$. Thus, the condition (2.5) becomes

$$
0<s<\sqrt{E} \text {. }
$$

As mentioned in section 1, we investigate linear stability as our first step. The linearized system of $(1.1)$ at the shock profile $(\bar{v}, \bar{u}, \bar{p})$ is the following system:

$$
\begin{gathered}
v_{t}-u_{x}=0 \\
u_{t}+p_{x}=0 \\
(p+E v)_{t}=-p+p_{R}(\bar{v})+p_{R}^{\prime}(\bar{v})(v-\bar{v}) .
\end{gathered}
$$

Consider (2.7) with the initial data:

$$
v(x, 0)=v_{0}(x), \quad u(x, 0)=u_{0}(x), \quad p(x, 0)=p_{0}(x) .
$$

Assume that

$$
\left|\int\left(v_{0}(x)-\bar{v}(x)\right) d x\right|, \mid \int\left(u_{0}(x)-\bar{u}(x) d x \mid<+\infty .\right.
$$

Then we can decompose them as follows:

$$
\begin{aligned}
& \left(\int\left(v_{0}(x)-\bar{v}(x)\right) d x, \int\left(u_{0}(x)-\bar{u}(x)\right) d x\right) \\
& \quad=x_{0}\left(v^{+}-v^{-}, u^{+}-u^{-}\right)+\delta\left(1, \sqrt{-p_{R}^{\prime}\left(v^{-}\right)}\right) .
\end{aligned}
$$

Suppose that the shock wave is sufficiently weak, i.e.,

$$
\varepsilon=:\left|v^{+}-v^{-}\right| \ll 1
$$

so that the vectors $\left(v^{+}-v^{-}, u^{+}-u^{-}\right)^{\mathrm{T}}$ and $\left(1, \sqrt{-p_{R}^{\prime}\left(v^{-}\right)}\right)^{\mathrm{T}}$ are linearly independent (noting that the vector $\left(1, \sqrt{-p_{R}^{\prime}\left(v^{-}\right)}\right)^{\mathrm{T}}$ is the right eigenvector belonging to the 1family). Thus, the translation $x_{0}$ of the shock profile and $\delta$ can be determined uniquely. We decompose the solution as follows:

$$
\begin{aligned}
& v(x, t)=\bar{v}\left(x+x_{0}, t\right)+\frac{a}{\sqrt{t+1}} m(x, t)+\frac{a_{1}}{t+1} m_{1}(x, t)+v^{*}, \\
& u(x, t)=\bar{u}\left(x+x_{0}, t\right)+\frac{b}{\sqrt{t+1}} m(x, t)+\frac{b_{1}}{t+1} m_{1}(x, t)+u^{*}, \\
& p(x, t)=\bar{p}\left(x+x_{0}, t\right)+\frac{c}{\sqrt{t+1}} m(x, t)+\frac{c_{1}}{t+1} m_{1}(x, t)+p^{*} .
\end{aligned}
$$

As mentioned in section 1, we hope that the new waves (the second and third terms in $\left.(2.12)_{1,2}\right)$ with base state $\left(v^{-}, u^{-}\right)$have some diffusive properties, move along a 1characteristic direction and carry the "excess mass" $\delta\left(1, \sqrt{-p_{R}^{\prime}\left(v^{-}\right)}\right)^{\mathrm{T}}$. Then we require $m(x, t)$ to have the form of

$$
m(z), \quad z=\frac{x-\lambda_{1}\left(v^{-}\right)(t+1)}{\sqrt{t+1}}
$$

such that

$$
\int m(z) d z=\delta
$$

where $\lambda_{1}\left(v^{-}\right)=-\sqrt{-p_{R}^{\prime}\left(v^{-}\right)}$. Similarly, we let $m_{1}(x, t)=m_{1}(z)$. 
A natural choice of $a, b, c$ is

$$
a=1, \quad b=\sqrt{-p_{R}^{\prime}\left(v^{-}\right)}=-\lambda_{1}\left(v^{-}\right)=:-\lambda_{1}, \quad c=p_{R}^{\prime}\left(v^{-}\right)=-\lambda_{1}^{2} .
$$

Inserting (2.12) into (2.7), noting (2.15), we arrive at

$$
\begin{gathered}
v_{t}^{*}-u_{x}^{*}=\frac{1}{(t+1)^{3 / 2}}\left(\frac{1}{2} m+\frac{1}{2} z m^{\prime}+\lambda_{1} a_{1} m_{1}^{\prime}+b_{1} m_{1}^{\prime}\right)+\frac{1}{(t+1)^{2}}\left(a_{1} m_{1}+\frac{1}{2} z a_{1} m_{1}^{\prime}\right) \\
u_{t}^{*}+p_{x}^{*}=\frac{1}{(t+1)^{3 / 2}}\left(\frac{1}{2} b m+\frac{1}{2} b z m^{\prime}+\lambda_{1} b_{1} m_{1}^{\prime}-c_{1} m_{1}^{\prime}\right)+\frac{1}{(t+1)^{2}}\left(b_{1} m_{1}+\frac{1}{2} z b_{1} m_{1}^{\prime}\right), \\
\left(p_{t}^{*}+E v^{*}\right)_{t}=-p^{*}+p_{R}^{\prime}(\bar{v}) v^{*}+\frac{1}{(t+1)^{1 / 2}}\left(p_{R}^{\prime}(\bar{v})-p_{R}^{\prime}\left(v^{-}\right)\right) m \\
+\frac{1}{(t+1)}\left(p_{R}^{\prime}(\bar{v}) a_{1} m_{1}-c_{1} m_{1}+\lambda_{1} c m^{\prime}+E \lambda_{1} m^{\prime}\right) \\
+\frac{1}{(t+1)^{3 / 2}}\left[\frac{1}{2} c m+\frac{1}{2} c z m^{\prime}+\lambda_{1} c_{1} m_{1}^{\prime}+E\left(\frac{1}{2} m+\frac{1}{2} z m^{\prime}+\lambda_{1} a_{1} m_{1}^{\prime}\right)\right] \\
+\frac{1}{(t+1)^{2}}\left[c_{1} m_{1}+\frac{1}{2} z c_{1} m_{1}^{\prime}+E a_{1} m_{1}+\frac{1}{2} E a_{1} z m_{1}^{\prime}\right]
\end{gathered}
$$

where' denotes $d / d z$.

We hope

$$
\begin{aligned}
\frac{1}{2}(z m)^{\prime}+\left(\lambda_{1} a_{1}+b_{1}\right) m_{1}^{\prime} & =0 \\
\frac{1}{2} b(z m)^{\prime}+\left(\lambda_{1} b_{1}-c_{1}\right) m_{1}^{\prime} & =0 \\
p_{R}^{\prime}\left(v^{-}\right) a_{1} m_{1}-c_{1} m_{1}+\lambda_{1}(c+E) m^{\prime} & =0 .
\end{aligned}
$$

Take $a_{1}=1$ and $c_{1}=0$. Thus,

$$
\begin{aligned}
\frac{1}{2} z m+\left(\lambda_{1}+b_{1}\right) m_{1} & =0, \\
-\frac{1}{2} \lambda_{1} z m+\lambda_{1} b_{1} m_{1} & =0 .
\end{aligned}
$$

Next we choose $b_{1}=-\frac{1}{2} \lambda_{1}$. As a result,

$$
m_{1}=-\lambda_{1}^{-1} z m,
$$

which together with (2.21) yields that

$$
p_{R}^{\prime}\left(v^{-}\right) m_{1}+\lambda_{1}\left(E+p_{R}^{\prime}\left(v^{-}\right)\right) m^{\prime}=0,
$$

i.e.,

$$
d m^{\prime}+z m=0
$$

where $d=E+p_{R}^{\prime}\left(v^{-}\right)$.

As a stability criterion in $[\mathrm{LI}]$, the following subcharacteristic condition should be satisfied, namely,

$$
E+p_{R}^{\prime}(v)>0
$$


for

$$
v^{-} \leq v \leq v^{+}
$$

especially,

$$
d>0 \text {. }
$$

The combination of (2.23) with (2.14) gives

$$
m(z)=\frac{\delta}{\sqrt{2 \pi d}} \exp \left(-\frac{z^{2}}{2 d}\right)
$$

We get from (2.22) that

$$
m_{1}(z)=\lambda_{1}^{-1} d m^{\prime}
$$

since

$$
z m=-d m^{\prime} .
$$

Thus,

$$
\int m_{1}(z) d z=0
$$

which means that the high-order correction carries zero mass. Then we conclude that

$$
\begin{aligned}
v_{t}^{*}-u_{x}^{*}= & \frac{1}{2} d \lambda_{1}^{-1}(t+1)^{-2}(z m)_{z z}=\frac{1}{2} d \lambda_{1}^{-1}(t+1)^{-1}(z m)_{x x}, \\
u_{t}^{*}+p_{x}^{*}= & -\frac{1}{4} d(t+1)^{-2}(z m)_{z z}=-\frac{1}{4} d(t+1)^{-1}(z m)_{x x}, \\
\left(p^{*}+E v^{*}\right)_{t}= & -p^{*}+p_{R}^{\prime}(\bar{v}) v^{*}+(t+1)^{-1 / 2}\left(p_{R}^{\prime}(\bar{v})-p_{R}^{\prime}\left(v^{-}\right)\right)\left(m+d \lambda_{1}^{-1} m_{x}\right) \\
& +\frac{1}{2}(t+1)^{-1}\left[(z m)_{x}+d E \lambda_{1}^{-1}(z m)_{x x}\right] .
\end{aligned}
$$

Due to (2.10) and the fact that

$$
\begin{aligned}
& \int\left(\bar{v}\left(x+x_{0}\right)-\bar{v}(x)\right) d x=x_{0}\left(v^{+}-v^{-}\right), \\
& \int\left(\bar{u}\left(x+x_{0}\right)-\bar{u}(x)\right) d x=x_{0}\left(u^{+}-u^{-}\right),
\end{aligned}
$$

we have

$$
\int v^{*}(x, 0) d x=0, \quad \int u^{*}(x, 0) d x=0 .
$$

Hence, the conservation form of (2.30) and (2.31) leads to

$$
\int v^{*}(x, t) d x=0, \quad \int u^{*}(x, t) d x=0
$$

for all $t \geq 0$. We will work on Eqs. (2.30)-(2.32) in Sec. 3 .

The following estimates on the shock profile will play an important role in our stability analysis. 
LEMMA 2.1. If (2.1)-(2.6) hold, then

$$
\begin{gathered}
0<\bar{v}_{y}(y)<C_{1} \varepsilon \exp \left(-C_{2}|y|\right) \\
\left|\bar{v}_{y y}(y)\right|,\left|\bar{v}_{y y y}(y)\right|<C_{3} \varepsilon \exp \left(-C_{4}|y|\right)
\end{gathered}
$$

where $C_{1}(i=1,2,3,4)$ is a positive constant, here and below, and $\varepsilon=:\left|v^{+}-v^{-}\right|$.

Proof. From the definition of $\bar{v}(y)$, it follows that

$$
\frac{d \bar{v}(y)}{d y}=\frac{-s^{2}\left(\bar{v}(y)-v^{-}\right)+p^{-}-p_{R}(\bar{v})}{s\left(E-s^{2}\right)}=-\frac{s^{2}\left(\bar{v}(y)-v^{-}\right)+p_{R}(\bar{v})-p_{R}\left(v^{-}\right)}{s\left(E-s^{2}\right)} .
$$

It has been proved in [HL] that $v^{-} \leq \bar{v}(y) \leq v^{+}$. Then $\bar{v}_{y}(y)>0$ is implied by (2.2) and (2.6). Thus $v^{-}<\bar{v}(y)<v^{+}$. We claim that the entropy condition B implies the Lax shock condition due to the convexity of $p_{R}$, namely,

$$
-p_{R}^{\prime}\left(v^{+}\right)<s^{2}<-p_{R}^{\prime}\left(v^{-}\right)
$$

In fact,

$$
-\frac{p_{R}(v)-p_{R}\left(v^{-}\right)}{v-v^{-}}=\int_{0}^{1}-p_{R}^{\prime}\left(v^{-}+r\left(v-v^{-}\right)\right) d r<-p_{R}^{\prime}\left(v^{-}\right)
$$

for $v^{-}<v<v^{+}$. Hence, $s^{2}<-p_{R}^{\prime}\left(v^{-}\right)$, and similarly we obtain $s^{2}>-p_{R}^{\prime}\left(v^{+}\right)$.

Thus, there exists $y_{0} \in R^{1}$ such that $s^{2}=-p_{R}^{\prime}\left(\bar{v}\left(y_{0}\right)\right)$. Without loss of generality, we can assume that $y_{0}=0$. Then

$$
s^{2}+p_{R}^{\prime}(\bar{v}(y))<0, \quad y<0
$$

and

$$
s^{2}+p_{R}^{\prime}(\bar{v}(y))>0, \quad y>0 .
$$

Moreover, $p_{R}^{\prime}(\bar{v}(y))$ is strictly increasing with respect to $y$ since $p_{R}^{\prime \prime}(v)>0$ for $v^{-}<v<$ $v^{+}$and $\bar{v}_{y}(y)>0$ for $y \in R$. Differentiation of (2.37) with respect to $y$ yields

$$
\frac{d \bar{v}_{y}(y)}{d y}=-\frac{\left(s^{2}+p_{R}^{\prime}(\bar{v}(y)) \bar{v}_{y}(y)\right.}{s\left(E-s^{2}\right)}
$$

Hence, we see

$$
\bar{v}_{y}(y)=v_{y}(0) \exp \left(-\int_{0}^{y} \frac{\left(s^{2}+p_{R}^{\prime}(\bar{v}(y))\right)}{s\left(E-s^{2}\right)} d y\right) .
$$

It is easy to get from $(2.37)$ that

$$
0<\bar{v}_{y}(y)<C \varepsilon
$$

for $y \in R$ and some positive constant $C$.

A combination of (2.38), (2.40), and (2.41) gives (2.35). The bounds (2.36) can be obtained easily from (2.35) and (2.37), which finishes the proof of Lemma 2.1.

REMARK 2.1. $n(x, t)=:(t+1)^{-1 / 2} m(x, t)$ solves the following equation:

$$
n_{t}+\lambda_{1}\left(v^{-}\right) n_{x}=\left(E+p_{R}^{\prime}\left(v^{-}\right)\right) n_{x x} .
$$


When one studies nonlinear stability, $n$ can be constructed as the solution of the following equation:

$$
n_{t}+\lambda_{1}\left(v^{-}\right) n_{x}+\frac{1}{2} \nabla \lambda_{1} \cdot r_{1}\left(v^{-}\right)=\left(E+p_{R}^{\prime}\left(v^{-}\right)\right) n_{x x}
$$

where $r_{1}$ is the right eigenvector belonging to the 1-family.

3. Stability analysis and the main result. Our main goal is to show that the solution to $(2.7),(2.8)$ converges asymptotically in time to the shock profile. It is stated in the following Theorem 3.1 .

Theorem 3.1. Suppose the function $p_{R}$ satisfies the condition H1) and that the subcharacteristic condition (2.24) is satisfied. Let $(\bar{v}, \bar{u}, \bar{p})\left(x+x_{0}-s t\right)$ be the shock profile of (1.1), and let $x_{0}$ be the translation uniquely determined through (2.10). Suppose that the initial data $\left(v_{0}, u_{0}, p_{0}\right)(x)$ satisfies $\left(v_{0}-\bar{v}, u_{0}-\bar{u}, p_{0}-\bar{p}\right) \in H^{1}(R)$ and that

$$
\begin{aligned}
& \int\left(1+x^{2}\right)\left(v_{0}(x)-\bar{v}\left(x+x_{0}\right)\right)^{2} d x<+\infty \\
& \int\left(1+x^{2}\right)\left(u_{0}(x)-\bar{u}\left(x+x_{0}\right)\right)^{2} d x<+\infty
\end{aligned}
$$

If the shock is weak, i.e., $\varepsilon=:\left|v^{+}-v^{-}\right|$is suitably small, then it follows that

$$
\begin{gathered}
\lim _{t \rightarrow \infty} \int\left(w(x, t)-\bar{w}\left(x-s t+x_{0}\right)\right)^{2} d x=0 \\
\lim _{t \rightarrow \infty} \sup _{x \in R^{1}}\left|w(x, t)-\bar{w}\left(x-s t+x_{0}\right)\right|=0
\end{gathered}
$$

for the smooth solution $w(x, t)=:(v, u, p)(x, t)$ of $(2.7),(2.8)$ and the shock profile $\bar{w}=:(\bar{v}, \bar{u}, \bar{p})$.

Before the proof of Theorem 3.1, we reset the problem on the moving coordinate $(y, t)$ with $y=x+x_{0}-s t$. It is natural to let

$$
V(y, t)=\int_{-\infty}^{y} v^{*}(r, t) d r, \quad U(y, t)=\int_{-\infty}^{y} u^{*}(r, t) d r
$$

due to (2.34). Thus,

$$
V( \pm \infty, t)=U( \pm \infty, t)=0
$$

We also let $P(y, t)=p(y, t)$. From (2.30)-(2.32) we see that $(V, U, P)$ satisfies the following equations:

$$
\begin{aligned}
& V_{t}-s V_{y}-U_{y}=\frac{1}{2} d \lambda_{1}^{-1}(t+1)^{-1}(z m)_{y} \\
& U_{t}-s U_{y}+P=-\frac{1}{4} d(t+1)^{-1}(z m)_{y}
\end{aligned}
$$




$$
\begin{aligned}
(P+ & \left.E V_{y}\right)_{t}-s\left(P+E V_{y}\right)_{y}=-P+p_{R}^{\prime}(\bar{v}) V_{y} \\
& +(t+1)^{-1 / 2}\left(p_{R}^{\prime}(\bar{v})-p_{R}^{\prime}\left(v^{-}\right)\right)\left(m+d \lambda_{1}^{-1} m_{y}\right) \\
& +\frac{1}{2}(t+1)^{-1}\left((z m)_{y}+E d \lambda_{1}^{-1}(z m)_{y y}\right) .
\end{aligned}
$$

Equations (3.6)-(3.8) give

$$
\begin{aligned}
& P_{t}-s P_{y}+E U_{y y}+P-p_{R}^{\prime}(\bar{v}) V_{y} \\
& \quad=(t+1)^{-1 / 2}\left(P_{R}^{\prime}(\bar{v})-p_{R}^{\prime}\left(v^{-}\right)\right)\left(m+d \lambda_{1}^{-1} m_{y}\right)+\frac{1}{2}(t+1)^{-1}(z m)_{y} .
\end{aligned}
$$

The first term in the right-hand side of (3.9) has a sufficiently large decay rate due to the different propagation speeds of shock and diffusive waves (see (3.12)).

The weighted Poincaré inequality (see $[\mathrm{HaL}]$, Th. 328) gives

$$
\begin{aligned}
& \left.\left|\int_{0}^{\infty}\right| \int_{y}^{\infty}\left(v_{0}(x)-\bar{v}\left(x+x_{0}\right)-m(x, 0)-m_{1}(x, 0)\right) d x\right|^{2} d y \mid \\
& \quad \leq 4 \int_{0}^{\infty}\left(v_{0}(x)-\bar{v}\left(x+x_{0}\right)-m(x, 0)-m_{1}(x, 0)\right)^{2} x^{2} d x
\end{aligned}
$$

Therefore, $V(y, 0)=V_{0}(y) \in H^{2}(R)$. Similarly $U(y, 0)=U_{0}(y) \in H^{2}(R)$ and we know that $P(y, 0) \in H^{1}(R)$.

In a routine manner, it is easy to know (at least for a weak shock) that (3.6)-(3.8) with initial data $\left(V_{0}(y), U_{0}(y), P_{0}(y)\right)$ admits a unique smooth solution $(V, U, P)$ satisfying $(V(\cdot, t), U(\cdot, t)) \in H^{2}(R), P(\cdot, t) \in H^{1}(R)$ for all $t \geq 0$ because (3.6)-(3.8) is a linear system.

This fact can also be proved by using Lemma 3.2.

To prove Theorem 3.1, it is sufficient to prove the following a priori estimates.

Lemma 3.2. Suppose $(V, U)(\cdot, t) \in H^{2}(R), P(\cdot, t) \in H^{1}(R)\left(0 \leq t \leq T_{0}\right)$ is the smooth solution of $(3.6)-(3.8)$ with the initial data $\left(V_{0}(y), U_{0}(y), P_{0}(y)\right)$. Then there exists a positive constant $\varepsilon_{0}$ such that if $\varepsilon=\left|v^{+}-v^{-}\right|<\varepsilon_{0}$, then it follows that

$$
\begin{aligned}
& \|V(\cdot, T)\|_{2}^{2}+\|U(\cdot, T)\|_{2}^{2}+\|P(\cdot, T)\|_{1}^{2} \\
& \quad+\int_{0}^{\mathrm{T}}\left(\left\|V_{y}(\cdot, t)\right\|_{1}^{2}+\left\|U_{y}(\cdot, t)\right\|_{1}^{2}+\|P(\cdot, t)\|_{1}^{2} d t\right. \\
& \quad \leq C\left[\|V(\cdot, 0)\|_{2}^{2}+\|U(\cdot, 0)\|_{2}^{2}+\|P(\cdot, 0)\|_{1}^{2}\right]+C, \quad 0 \leq T \leq T_{0} .
\end{aligned}
$$

Hereafter we use $C$ to denote a generic constant independent of $T$ and $T_{0}$ without confusion. Without loss of generality, we can assume that $x_{0}=0$ for convenience. We use $\|\cdot\|_{k}$ to denote the norm in $H^{k}$, and use $\|\cdot\|$ to denote the norm in $L_{2}$. 
Proof. At first, we have the following equation:

$$
\begin{aligned}
\frac{1}{2}\left\{E U_{y}^{2}\right. & \left.+P^{2}+\frac{-p_{R}^{\prime}(\bar{v})}{E+p_{R}^{\prime}(\bar{v})}\left(P+E V_{y}\right)^{2}\right\}_{t}+\frac{E\left(P-p_{R}^{\prime}(\bar{v}) V_{y}\right)^{2}}{E+p_{R}^{\prime}(\bar{v})} \\
= & \{\cdots\}_{y}-\frac{1}{4} E d(t+1)^{-1}(z m)_{y y} U_{y}-\frac{s}{2}\left(P+E V_{y}\right)^{2}\left\{\frac{-p_{R}^{\prime}(\bar{v})}{E+p_{R}^{\prime}(\bar{v})}\right\}_{y} \\
& +\frac{E\left(P-p^{\prime}(\bar{v}) V_{y}\right)}{E+p_{R}^{\prime}(\bar{v})}(t+1)^{-1 / 2}\left(p_{R}^{\prime}(\bar{v})-p_{R}^{\prime}\left(v^{-}\right)\right)\left(m+d \lambda_{1}^{-1} m_{y}\right) \\
& +\frac{E\left(P-p^{\prime}(\bar{v}) V_{y}\right)}{2\left(E+p_{R}^{\prime}(\bar{v})\right)}(t+1)^{-1}(z m)_{y} \\
& +\frac{-p_{R}^{\prime}(\bar{v})}{2\left(E+p_{R}^{\prime}(\bar{v})\right)}\left(P+E V_{y}\right)(t+1)^{-1} E d \lambda_{1}^{-1}(z m)_{y y}
\end{aligned}
$$

where $\{\cdots\}_{y}$ denotes the term that disappears after integration with respect to $y \in R$. We claim that there exist positive constants $C_{i}(i=5,6)$ such that

$$
\int\left|\bar{v}(y)-v^{-}\right||m(y, t)| d y \leq C_{5} \varepsilon \exp \left(-C_{6}(t+1)\right)
$$

due to the different propagation speeds of the shock and diffusive waves. In fact, it follows from Lemma 2.1 that

$$
\begin{aligned}
\left|\bar{v}(y)-v^{-}\right| & \leq \int_{-\infty}^{y}\left|\bar{v}_{y}(r)\right| d r \\
& \leq C_{7} \varepsilon \exp \left(C_{8} y\right), \quad y<0
\end{aligned}
$$

and

$$
\left|\bar{v}(y)-v^{-}\right| \leq C_{9} \varepsilon
$$

for some positive constants $C_{i}(i=7,8,9)$.

We write $m(z)$ as

$$
m(y, t)=\frac{\delta}{\sqrt{2 d \pi}} \exp \left(-\frac{1}{2 d}\left(\frac{y+\left(s-\lambda_{1}\right)(t+1)-s}{\sqrt{t+1}}\right)^{2}\right) .
$$

Also, we note that $s-\lambda_{1}>0$ due to $\lambda_{1}<0$. It is easy to verify (3.12) by dividing the integral interval into $\left(-\infty,-\frac{1}{2}\left(s-\lambda_{1}\right)(t+1)\right),\left(-\frac{1}{2}\left(s-\lambda_{1}\right)(t+1), 0\right)$ and $(0,+\infty)$, and use (3.13), (3.14) on the different intervals.

A similar estimate holds when we use $m_{y}(y, t)$ instead of $m(y, t)$ in (3.12).

Integrate (3.11) over $R \times[0, T]$. Then Lemma 2.1, the explicit expression of $m$, the subcharacteristic condition (2.24), and the Cauchy-Schwarz inequality yield that

$$
\begin{aligned}
\| U_{y}(\cdot, T) & \left\|^{2}+\right\| P(\cdot, T)\left\|^{2}+\right\| V_{y}(\cdot, T)\left\|^{2}+\int_{0}^{\mathrm{T}}\right\|\left(P-p_{R}^{\prime}(v) V_{y}\right) \|^{2}(t) d t \\
\leq & C\left[\left\|U_{y}(\cdot, 0)\right\|^{2}+\|P(\cdot, 0)\|^{2}+\left\|V_{y}(\cdot, 0)\right\|^{2}\right] \\
& +C \ell \int_{0}^{\mathrm{T}}\left(\|P\|^{2}+\left\|V_{y}\right\|^{2}+\left\|U_{y}\right\|^{2}\right) d t \\
& +C \varepsilon \int_{0}^{\mathrm{T}}\left(\|P\|^{2}+\left\|V_{y}\right\|^{2}\right) d t+C\left(\ell^{-1}+1\right)
\end{aligned}
$$


where $\ell$ is an arbitrarily small positive number that will be determined later on.

It is convenient to write the equations of $(V, U, P)$ as

$$
\begin{gathered}
L_{1}(V, U)=\frac{1}{2} d \lambda_{1}^{-1}(t+1)^{-1}(z m)_{y}, \\
L_{2}(V, U)=(t+1)^{-1 / 2}\left(p_{R}^{\prime}(\bar{v})-p_{R}^{\prime}\left(v^{-}\right)\right)\left(m+d \lambda_{1}^{-1} m_{y}\right)+\frac{1}{2}(t+1)^{-1}(z m)_{y},
\end{gathered}
$$

where

$$
L_{1}(U, V)=V_{t}-s V_{y}-U_{y}
$$

and

$$
L_{2}(V, U)=P_{t}-s P_{y}+E U_{y y}+P+A^{-1} V_{y}
$$

with $A^{-1}=-p_{R}^{\prime}(\bar{v}), P=s U_{y}-U_{t}-\frac{1}{4} d(t+1)^{-1}(z m)_{y}$.

By an approach similar to that used in $[\mathrm{KM}],[\mathrm{HL}]$, and $[\mathrm{X}]$, we discuss the equation

$$
\begin{aligned}
& V L_{1}(V, U)-A U L_{2}(V, U)=\frac{1}{2} d \lambda_{1}^{-1}(t+1)^{-1}(z m)_{y} V \\
& \quad-(t+1)^{-1 / 2}\left(p_{R}^{\prime}(\bar{v})-p_{R}^{\prime}\left(v^{-}\right)\right)\left(m+d \lambda_{1}^{-1} m_{y}\right) A U \\
& \quad-\frac{1}{2}(t+1)^{-1}(z m)_{y} A U .
\end{aligned}
$$

The left-hand side is reduced to

$$
\begin{aligned}
\left\{\frac{1}{2} V^{2}+\frac{1}{2} A U^{2}-A U P+\right. & \left.\frac{1}{2} s A_{y} U^{2}\right\}_{t}+E A U_{y}^{2}+\frac{1}{2} s A_{y} U^{2}-A P^{2}+\left(E-s^{2}\right) A_{y} U U_{y} \\
& +\frac{1}{4}(t+1)^{-1} d(z m)_{y}\left(s A_{y} U+A U-A P\right)+\{\cdots\}_{y} .
\end{aligned}
$$

To dominate the term $\left(-A P^{2}\right)$, we calculate

$$
\begin{aligned}
U_{y} L_{1}( & V, U)+A P L_{2}(V, U) \\
= & \frac{1}{2} d \lambda_{1}^{-1}(t+1)^{-1}(z m)_{y} U_{y} \\
& +(t+1)^{-1 / 2}\left(p_{R}^{\prime}(\bar{v})-p_{R}^{\prime}\left(v^{-}\right)\right)\left(m+d \lambda_{1}^{-1} m_{y}\right) A P \\
& +\frac{1}{2}(t+1)^{-1}(z m)_{y} A P .
\end{aligned}
$$

The left-hand side is reduced to

$$
\begin{array}{r}
\left\{\frac{1}{2} E A U_{y}^{2}+\frac{1}{2} A P^{2}+U_{y} V\right\}_{t}+\left(A+\frac{1}{2} s A_{y}\right) P^{2}-\left(1+\frac{1}{2} s E A_{y}\right) U_{y}^{2}+E A_{y} U_{t} U_{y} \\
-\frac{1}{4} d(t+1)^{-1}(z m)_{y} V_{y}-\frac{1}{4} d(t+1)^{-1} E A U_{y y}(z m)_{y}+\{\cdots\}_{y} .
\end{array}
$$

Notice that $(3.20)+(3.22) \times k$ with a suitable positive number $k$ gives

$$
\begin{aligned}
\frac{1}{2} F_{t}+ & \left.\left\{E A-k\left(1+\frac{1}{2} s E A_{y}\right)\right\} U_{y}^{2}+\left[(k-1) A+\frac{1}{2} s A_{y}\right)\right] P^{2}+\frac{1}{2} s A_{y} U^{2} \\
& +\left(E-s^{2}\right) A_{y} U U_{y}+k E A_{y} U_{t} U_{y}+\{\cdots\}_{y} \\
= & \frac{1}{2} d \lambda_{1}^{-1}(t+1)^{-1}(z m)_{y}\left(V+k U_{y}\right)-\frac{1}{4} d(t+1)^{-1}(z m)_{y}\left\{s A_{y} U+A U\right. \\
& \left.+A P-V_{y}-E A U_{y y}\right\} \\
& +(t+1)^{-1 / 2}\left(p_{R}^{\prime}(\bar{v})-p_{R}^{\prime}\left(v^{-}\right)\right)\left(m+d \lambda_{1}^{-1} m_{y}\right)(A P-A U) \\
& +\frac{1}{2}(t+1)^{-1}(z m)_{y}(A P-A U),
\end{aligned}
$$


where

$$
F=V^{2}+A U^{2}-2 A U P+s A_{y} U^{2}+k E A U_{y}^{2}+k E A P^{2}+k U_{y} V .
$$

Since $E A>d_{1}>1$ for a positive constant $d_{1}$ due to the subcharacteristic condition (2.24), we can choose $k$ suitably such that, in view of Lemma 2.1,

$$
E A-k\left(1+\frac{1}{2} s E A_{y}\right)>d_{2}>0
$$

and

$$
(k-1) A+\frac{1}{2} s A_{y}>d_{3}>0
$$

for some constants $d_{2}, d_{3}$, if the shock is suitably weak (i.e., $\varepsilon$ is suitably small). We can write some terms appearing in (3.24) as follows:

$$
\begin{aligned}
(z m)_{y} V & =-(z m) V_{y}+\{\cdots\}_{y} \\
(z m)_{y} A U & =-(z m) A_{y} U-(z m) A U_{y}+\{\cdots\}_{y}, \\
(z m)_{y} A U_{y y} & =-(z m)_{y y} A U_{y}-(z m)_{y} A_{y} U_{y}+\{\cdots\}_{y} .
\end{aligned}
$$

The entropy condition (2.2) implies that

$$
s A_{y}>0 .
$$

We estimate each term in (3.24) as follows. It follows from the Cauchy-Schwarz inequality that

$$
\begin{gathered}
F \geq \frac{V^{2}}{2}+\left(\frac{A}{2}+s A_{y}\right) U^{2}+k E A U_{y}^{2}+K E A P^{2}-\left(2 A P^{2}+\frac{k^{2} U_{y}^{2}}{2}\right) \\
\left|\left(E-s^{2}\right) A_{y} U U_{y}\right| \leq \frac{1}{16} s A_{y} U^{2}+C U_{y}^{2} \\
\left|k E A_{y} U_{t} U_{y}\right| \\
=\left|k E A_{y}\left(s U_{y}-P-\frac{1}{4} d(t+1)^{-1}(z m)_{y}\right) U_{y}\right| \\
\leq C\left[U_{y}^{2}+P^{2}\right]+C(t+1)^{-2}\left[(z m)_{y}\right]^{2} .
\end{gathered}
$$

In view of (3.27)-(3.30) and the Cauchy-Schwarz inequality, we can arrive at

$$
\begin{gathered}
\left|\int_{0}^{T} \int \frac{1}{2} d \lambda_{1}^{-1}(t+1)^{-1}(z m)_{y}\left(V+k U_{y}\right) d y d t\right| \\
\leq \int_{0}^{T} \int \frac{1}{16} s A_{y} U^{2} d y d t+C \int_{0}^{T} \int\left(V_{y}^{2}+U_{y}^{2}\right) d y d t \\
+C \int_{0}^{T} \int(t+1)^{-2}\left[(z m)^{2}+\left[(z m)_{y}\right]^{2} d y d t\right. \\
\left|\int_{0}^{T} \int \frac{1}{4} d(t+1)^{-1}(z m)_{y}\left(s A_{y} U+A U-A P-V_{y}-E A U_{y y}\right) d y d t\right| \\
\leq \int_{0}^{T} \int \frac{1}{16} s A_{y} U^{2} d y d t+C \int_{0}^{T} \int\left(V_{y}^{2}+U_{y}^{2}+P^{2}\right) d y d t \\
+C \int_{0}^{T} \int(t+1)^{-2}\left[(z m)^{2}+\left[(z m)_{y}\right]^{2}+\left[(z m)_{y y}\right]^{2}\right] d y d t
\end{gathered}
$$




$$
\begin{aligned}
& \left|\int_{0}^{T} \int(t+1)^{-1 / 2}\left(p_{R}^{\prime}(\bar{v})-p_{R}^{\prime}\left(v^{-}\right)\right)\left(m+d \lambda_{1}^{-1} m_{y}\right)(A P-A U) d y d t\right| \\
& \leq C \int_{0}^{T} \int\left[(t+1)^{-1 / 2}\left(p_{R}^{\prime}(\bar{v})-p_{R}^{\prime}\left(v^{-}\right)\right)\left(m+d \lambda_{1}^{-1} m_{y}\right)\right]^{2} d y d t \\
& \quad+C \int_{0}^{T} R(t) d t+C \int_{0}^{T} \int P^{2} d y d t+C \int_{0}^{T} \sup _{y \in R^{1}}|U(y, t)| R(t) d t \\
& \leq C \int_{0}^{T} R(t) d t+C \int_{0}^{T} \int P^{2} d y d t+C \int_{0}^{T} \sup _{y \in R^{1}}|U(y, t)| R(t) d t
\end{aligned}
$$

where $R(t)=(t+1)^{-1 / 2} \int\left|\left(p_{R}^{\prime}(\bar{v})-p_{R}^{\prime}\left(v^{-}\right)\right)\left(m+d \lambda_{1}^{-1} m_{y}\right)\right|(y, t) d y$

$$
\begin{aligned}
& \left|\int_{0}^{T} \int \frac{1}{2}(t+1)^{-1}(z m)_{y}(A P-A U) d y d t\right| \\
& \leq \int_{0}^{T} \int \frac{1}{16} s A_{y} U^{2} d y d t+C \int_{0}^{T} \int\left(U_{y}^{2}+P^{2}\right) d y d t \\
& \quad+C \int_{0}^{T} \int(t+1)^{-2}\left[(z m)^{2}+\left[(z m)_{y}\right]^{2}\right] d y d t
\end{aligned}
$$

Thus, integration of $(3.24)$ over $[0, T) \times R^{1}$ and the above inequalities imply that

$$
\begin{aligned}
\|V(\cdot, T)\|^{2} & +\|U(\cdot, T)\|^{2}+\left\|U_{y}(\cdot, T)\right\|^{2}+\|P(\cdot, T)\|^{2} \\
& +\int_{0}^{T}\left(\left\|U_{y}(\cdot, t)\right\|^{2}+\|P(\cdot, t)\|^{2}\right) d t+\int_{0}^{T} \int s A_{y} U^{2} d y d t \\
\leq & \left(\|V(\cdot, 0)\|^{2}+\|U(\cdot, 0)\|^{2}+\left\|U_{y}(\cdot, 0)\right\|^{2}+\|P(\cdot, 0)\|^{2}\right. \\
& +C \int_{0}^{T}\left(\left\|U_{y}(\cdot, t)\right\|^{2}+\|P(\cdot, t)\|^{2}+\left\|V_{y}(\cdot, t)\right\|^{2}\right) d t \\
& +C \int_{0}^{T} R(t) d t+C \int_{0}^{T} \sup _{y \in R^{1}}|U(y, t)| R(t) d t \\
& +C \int_{0}^{T} \int(t+1)^{-2}\left[(z m)^{2}+\left[(z m)_{y}\right]^{2}+\left[(z m)_{y y}\right]^{2} d y d t\right.
\end{aligned}
$$

From the explicit expression of $m$, we see that the last term on the right-hand side of (3.37) can be bounded uniformly for all $T \geq 0$. Inequality (3.12) and its analogue by using $m_{y}$ instead of $m$ imply

$$
\int_{0}^{T} R(t) d t \leq C
$$


A combination of (3.37) and (3.15) gives

$$
\begin{aligned}
\|V(\cdot, T)\|_{1}^{2} & +\|U(\cdot, T)\|_{1}^{2}+\|P(\cdot, T)\|^{2} \\
& +\int_{0}^{T}\left(\left\|U_{y}(\cdot, t)\right\|^{2}+\|P(\cdot, t)\|^{2}\right) d t+\int_{0}^{T} \int s A_{y} U^{2} d y d t \\
& +\int_{0}^{T}\left\|P-p_{R}^{\prime}(\bar{v}) V_{y}\right\|^{2}(t) d t \\
\leq C & \left(\|V(\cdot, 0)\|_{1}^{2}+\|U(\cdot, 0)\|_{1}^{2}+\| P(\cdot, 0)\right) \|^{2} \\
& +C \ell \int_{0}^{T}\left(\left\|U_{y}(\cdot, t)\right\|^{2}+\| P\left((\cdot, t)\left\|^{2}+\right\| V_{y}(\cdot, t) \|^{2}\right) d t\right. \\
& +C \varepsilon \int_{0}^{T}\left(\|P(\cdot, t)\|^{2}+\left\|V_{y}(\cdot, t)\right\|^{2}\right) d t \\
& +C\left(\ell^{-1}+1\right)+C \int_{0}^{T} \sup _{y \in R^{1}}|U(y, t)| R(t) d t .
\end{aligned}
$$

Due to $\mathrm{H} 1$ ), we have

$$
0<b_{1} \leq p_{R}^{\prime}(\bar{v}) \leq b_{2}
$$

for some constants $b_{1}, b_{2}$. Thus

$$
\begin{aligned}
(P- & \left.p_{R}^{\prime}(\bar{v}) V_{y}\right)^{2} \\
& =P^{2}-2 p_{R}^{\prime}(\bar{v}) P V_{y}+\left[p_{R}^{\prime}(\bar{v})\right]^{2} V_{y}^{2} \\
& \geq P^{2}-\frac{3}{2} P^{2}-\frac{2}{3}\left[p_{R}^{\prime}(\bar{v})\right]^{2} V_{y}^{2}+\left[p_{R}^{\prime}(\bar{v})\right]^{2} V_{y}^{2} \\
& \geq-\frac{1}{2} P^{2}+\frac{1}{3} b_{1}^{2} V_{y}^{2} .
\end{aligned}
$$

Thus, we can choose $\ell$ suitably such that it follows, for small $\varepsilon$, that

$$
\begin{aligned}
\|V(\cdot, T)\|_{1}^{2} & +\|U(\cdot, T)\|_{1}^{2}+\|P(\cdot, T)\|^{2} \\
& +\int_{0}^{T}\left(\left\|U_{y}(\cdot, t)\right\|^{2}+\left\|V_{y}(\cdot, t)\right\|^{2}+\|P(\cdot, t)\|^{2}\right) d t+\int_{0}^{T} \int s A_{y} U^{2} d y d t \\
\leq C & \left(\|V(\cdot, 0)\|_{1}^{2}+\|U(\cdot, 0)\|_{1}^{2}+\|P(\cdot, 0)\|^{2}+C\right. \\
& +C \int_{0}^{T} \sup _{y \in R^{1}}|U(y, t)| R(t) d t .
\end{aligned}
$$

We express the last term in (3.42) by $Q$. Then it follows from Young's inequality that

$$
\begin{aligned}
Q \leq & C \int_{0}^{T}\|U\|^{1 / 2}\left\|U_{y}\right\|^{1 / 2} R(t) d t \\
& \leq \frac{1}{2} \int_{0}^{T}\left\|U_{y}\right\|^{2} d t+C \int_{0}^{T}\|U\|^{2 / 3}\|[R(t)]\|^{4 / 3} d t \\
& \leq \frac{1}{2} \int_{0}^{T}\left\|U_{y}\right\|^{2} d t+C \int_{0}^{T}\left(\|U\|^{2}+1\right)[R(t)]^{4 / 3} d t
\end{aligned}
$$


where the following inequality is used:

$$
U^{2}(y, t)=2 \int_{-\infty}^{y} U U_{y}(\theta, t) d \theta \leq 2\|U(\cdot, t)\|\left\|U_{y}(\cdot, t)\right\| .
$$

Similarly to (3.38), we have

$$
\int_{0}^{T}[R(t)]^{4 / 3} d t \leq C .
$$

From the above inequalities and Gronwall's inequality, we can conclude that

$$
\begin{aligned}
& \|V(\cdot, T)\|_{1}^{2}+\|U(\cdot, T)\|_{1}^{2}+\|P(\cdot, T)\|^{2} \\
& \quad+\int_{0}^{T}\left(\left\|U_{y}(\cdot, t)\right\|^{2}+\left\|V_{y}(\cdot, t)\right\|^{2}+\|P(\cdot, t)\|^{2}\right) d t+\int_{0}^{T} \int s A_{y} U^{2} d y d t \\
& \quad \leq C\left(\|V(\cdot, 0)\|_{1}^{2}+\|U(\cdot, 0)\|_{1}^{2}+\|P(\cdot, 0)\|^{2}+C .\right.
\end{aligned}
$$

The next step is to estimate the high-order derivatives. Using a similar method to that used in [HL], we differentiate $L_{1}(V, U)$ and $L_{2}(V, U)$ with respect to $y$ respectively. After that, we calculate

$$
V_{y} \partial_{y} L_{1}(V, U)-A U_{y} \partial_{y} L_{2}(V, U)
$$

and

$$
U_{y y} \partial_{y} L_{1}(V, U)+A P_{y} \partial_{y} L_{2}(V, U)
$$

Then (3.45), the Cauchy-Schwarz inequality, and Lemma 2.1 give

$$
\begin{aligned}
& \left\|U_{y y}(\cdot, T)\right\|^{2}+\left\|P_{y}(\cdot, T)\right\|^{2}+\int_{0}^{T}\left(\left\|P_{y}(\cdot, t)\right\|^{2}+\left\|U_{y y}(\cdot, t)\right\|^{2} d t\right. \\
& \leq C q \int_{0}^{T}\left\|V_{y y}(\cdot, t)\right\|^{2} d t+C\left(q^{-1}+1\right)
\end{aligned}
$$

if the shock is weak, where $q$ is an arbitrary positive number that arises when we use the Cauchy-Schwarz inequality, which will be chosen suitably later. Here we omit the details of the proof of (3.46) because we can get it by a similar method as in getting (3.45).

Let us consider the following equation:

$$
\begin{aligned}
& \left(P_{y}+E V_{y y}\right) \partial_{y y} L_{1}(V, U)+V_{y y} \partial_{y} L_{2}(V, U) \\
& =\frac{1}{2} d \lambda_{1}^{-1}(t+1)^{-1}\left(P_{y}+E V_{y y}\right)(z m)_{y y y} \\
& \quad+V_{y y}(t+1)^{-1 / 2}\left\{\left[p_{R}^{\prime}(\bar{v})-p_{R}^{\prime}\left(v^{-}\right)\right]\left(m+d \lambda_{1}^{-1} m_{y}\right)\right\}_{y}+\frac{1}{2} V_{y y}(t+1)^{-1}(z m)_{y y}
\end{aligned}
$$

The left-hand side of (3.47) can be expressed as

$$
\begin{gathered}
\frac{E}{2}\left(V_{y y}^{2}\right)_{t}-\frac{1}{2}\left(U_{y y}^{2}\right)_{t}+\left(V_{y y} P_{y}\right)_{t}+P_{y} V_{y y}+\left(A^{-1}\right)_{y} V_{y} V_{y y} \\
+A^{-1} V_{y y}^{2}+\{\cdots\}_{y}-\frac{1}{4} d(t+1)^{-1}(z m)_{y y y} U_{y y} .
\end{gathered}
$$


Integration of (3.48) gives, in view of (3.40),

$$
\begin{aligned}
& \frac{E}{2}\left\|V_{y y}(\cdot, T)\right\|^{2}+b_{1} \int\left\|V_{y y}(\cdot, t)\right\|^{2} d t \\
& \leq \frac{E}{2}\left\|V_{y y}(\cdot, 0)\right\|^{2}+\frac{1}{2}\left\|U_{y y}(\cdot, T)\right\|^{2}-\frac{1}{2}\left\|U_{y y}(\cdot, 0)\right\|^{2} \\
& \quad-\int V_{y y} P_{y}(y, T) d y+\int V_{y y} P_{y}(y, 0) d y-\int_{0}^{T} \int\left(V_{y y} P_{y}+\left(A^{-1}\right)_{y} V_{y} V_{y y}\right) d y d t \\
& \quad+\int_{0}^{T} \int\left\{\frac{1}{4} d(t+1)^{-1}(z m)_{y y y} U_{y y}\right. \\
& \quad+\int_{0}^{T} \int V_{y y}(t+1)^{-1 / 2}\left\{\left[p_{R}^{\prime}(\bar{v})-p_{R}^{\prime}\left(v^{-}\right)\right]\left(m+d \lambda_{1}^{-1} m_{y}\right)\right\}_{y} d y d t \\
& \quad+\int_{0}^{T} \int V_{y y}(t+1)^{-1}(z m)_{y y} d y d t .
\end{aligned}
$$

With a similar approach to that used to get (3.12), one can obtain that

$$
\int\left(\left\{\left[p_{R}^{\prime}(\bar{v})-p_{R}^{\prime}\left(v^{-}\right)\right]\left(m+d \lambda_{1}^{-1} m_{y}\right)\right\}_{y}\right)^{2} d y \leq C_{10} \varepsilon \exp \left(-C_{11}(t+1)\right) .
$$

Each term in (3.49) can be estimated with the help of the Cauchy-Schwarz inequality, (3.45), (3.50), and the explicit expression of $m$. After that, we can arrive at

$$
\begin{aligned}
& \left\|V_{y y}(\cdot, T)\right\|^{2}+\int_{0}^{T}\left\|V_{y y}(\cdot, t)\right\|^{2} d t \\
& \quad \leq\left\|P_{y}(\cdot, T)\right\|^{2}+\left\|U_{y y}(\cdot, T)\right\|^{2}+\int_{0}^{T}\left(\left\|P_{y}(\cdot, t)\right\|^{2}+\left\|U_{y y}(\cdot, t)\right\|^{2}\right) d t+C,
\end{aligned}
$$

which together with (3.46) enable us to get, by choosing a suitable positive constant $q$, that

$$
\begin{aligned}
& \left\|V_{y y}(\cdot, T)\right\|^{2}+\left\|U_{y y}(\cdot, T)\right\|^{2}+\left\|P_{y}(\cdot, T)\right\|^{2} \\
& \quad+\int_{0}^{T}\left(\left\|V_{y y}(\cdot, t)\right\|^{2}+\left\|U_{y y}(\cdot, t)\right\|^{2}+\left\|P_{y}(\cdot, t)\right\|^{2}\right) d t \\
& \leq C,
\end{aligned}
$$

which completes the proof of Lemma 3.2.

We turn to the proof of Theorem 3.1. The global existence of a smooth solution for (3.6) $-(3.8)$ is standard, in view of the a priori estimates (3.10). From (3.6), (3.10), and the explicit expression of $m$, we have

$$
\int_{0}^{+\infty}\left(\left\|V_{y}(\cdot, t)\right\|^{2}+\left|\frac{d}{d t}\left\|V_{y}(\cdot, t)\right\|^{2}\right|\right)<\infty
$$


which implies that

$$
\lim _{t \rightarrow \infty}\left\|V_{y}(\cdot, t)\right\|^{2} \rightarrow 0
$$

Similarly, we can get

$$
\begin{gathered}
\lim _{t \rightarrow \infty}\left\|U_{y}(\cdot, t)\right\|^{2} \rightarrow 0 \\
\lim _{t \rightarrow \infty}\|P(\cdot, t)\|^{2} \rightarrow 0 .
\end{gathered}
$$

For any $(y, t) \in R^{1} \times R^{+}$, we have

$$
V_{y}(y, t)^{2}=2 \int_{-\infty}^{y} V_{y}(\theta, t) V_{y y}(\theta, t) d \theta \leq 2\left\|V_{y}(\cdot, t)\right\| \cdot\left\|V_{y y}(\cdot, t)\right\| .
$$

Hence, (3.53) and (3.54) imply

$$
\sup _{y \in R^{1}}\left|V_{y}(y, t)\right| \rightarrow 0, \quad t \rightarrow \infty .
$$

The same arguments give

$$
\begin{aligned}
& \sup _{y \in R^{1}}\left|U_{y}(y, t)\right| \rightarrow 0, \quad t \rightarrow \infty, \\
& \sup _{y \in R^{1}}|P(y, t)| \rightarrow 0, \quad t \rightarrow \infty .
\end{aligned}
$$

The explicit expression of $m$ and the above arguments lead to (3.3), (3.4), which completes the proof of Theorem 3.1.

Acknowledgment. The first author is grateful to Prof. L. Hsiao for her constant support, and also to the referee for the very helpful suggestions.

\section{REFERENCES}

[CE] C. Cercignani, The Boltzmann Equation and its Applications, Springer-Verlag, New York, 1998

[CLL] G. Q. Chen, C. D. Levermore, and T. P. Liu, Hyperbolic conservation laws with stiff relaxation terms and entropy, Comm. Pure Appl. Math. 47, 787-830 (1994)

[CP] R. E. Caflisch and G. C. Papanicolaou, The fluid dynamical limit of a nonlinear model Boltzmann equation, Comm. Pure Appl. Math. 32, 589-616 (1979)

[GO] J. Goodman, Nonlinear asymptotic stability of viscous shock profiles for conservation laws, Arch. Rational Mech. Anal. 95, 325-344 (1986)

[HaL] G. H. Hardy, J. E. Littlewood, and G. Polya, Inequalities, Cambridge University Press, 1934

[HL] L. Hsiao and T. Luo, The stability of travelling wave solutions for a rate type viscoelastic system, to appear

[IN] K. Inoue and T. Nishida, On the Broadwell model of the Boltzmann equation for a simple discrete velocity gas, Appl. Math. Optimization 3, 27-49 (1976)

[KM] S. Kawashima and A. Matsumura, Asymptotic stability to travelling wave solutions of systems for one-dimensional gas motion, Comm. Math. Phys. 101, 92-127 (1985)

[LI] T. P. Liu, Hyperbolic conservation laws with relaxation, Comm. Math. Phys. 108, 153-175 (1987)

[LIU] T. P. Liu, Nonlinear stability of shock waves for viscous conservation laws, Memoirs Amer. Math. Soc. 328 (1986)

[MN] A. Matsumura and K. Nishihara, On the stability of travelling wave solutions of a one-dimensional model system for compressible viscous gas, Japan J. Appl. Math. 2, 17-25 (1985)

[NA] R. Natalini, Convergence to the equilibrium solutions for the system of conservation laws with relaxation, preprint 
[PI] T. Platkowski and R. Illner, Discrete velocity models of the Boltzmann equation: a survey of the mathematical aspects of the theory, SIAM Review 30, 213-255 (1988)

[ST] M. Slemlod and A. E. Tzavaras, Self-similar fluid-dynamic limits for the Broadwell system, Arch. Rational Mech. Anal. 122, 353-392 (1993)

[SU] I. Suliciu, On modelling phase transitions by means of rate-type constitutive equations. Shock wave structure, Internat. J. Engrg. Sci. 28, 829-841 (1990)

[SX] A. Szepessy and Z. Xin, Nonlinear stability of viscous shock waves, Arch. Rational Mech. Anal. 122, 53-103 (1993)

[X] Z. Xin, The fluid-dynamic limit of the Broadwell model of the nonlinear Boltzmann equation in the presence of shocks, Comm. Pure Appl. Math. 44, 679-713 (1991) 